\title{
Article \\ Genome Sequence Analysis of the Oleaginous Yeast, Rhodotorula diobovata, and Comparison of the Carotenogenic and Oleaginous Pathway Genes and Gene Products with Other Oleaginous Yeasts
}

\author{
Irene Fakankun ${ }^{1}$, Brian Fristensky ${ }^{2}$ and David B. Levin ${ }^{1, *}$ \\ 1 Department of Biosystems Engineering, University of Manitoba, Winnipeg, MB R3T 2N2, Canada; \\ fakankui@myumanitoba.ca \\ 2 Department of Plant Science, University of Manitoba, Winnipeg, MB R3T 2N2, Canada; \\ brian.fristensky@umanitoba.ca \\ * Correspondence: david.levin@umanitoba.ca; Tel.: +1-204-474-7429; Fax: +1-204-474-7512
}

\section{check for}

updates

Citation: Fakankun, I.; Fristensky, B.; Levin, D.B. Genome Sequence Analysis of the Oleaginous Yeast, Rhodotorula diobovata, and Comparison of the Carotenogenic and Oleaginous Pathway Genes and Gene Products with Other Oleaginous Yeasts. J. Fungi 2021, 7, 320. https:// doi.org/10.3390/jof7040320

Academic Editors: Isabel Sa-Correia and Naseem A. Gaur

Received: 22 March 2021

Accepted: 16 April 2021

Published: 20 April 2021

Publisher's Note: MDPI stays neutral with regard to jurisdictional claims in published maps and institutional affiliations.

Copyright: (c) 2021 by the authors. Licensee MDPI, Basel, Switzerland. This article is an open access article distributed under the terms and conditions of the Creative Commons Attribution (CC BY) license (https:/ / creativecommons.org/licenses/by/ $4.0 /)$.
Abstract: Rhodotorula diobovata is an oleaginous and carotenogenic yeast, useful for diverse biotechnological applications. To understand the molecular basis of its potential applications, the genome was sequenced using the Illumina MiSeq and Ion Torrent platforms, assembled by AbySS, and annotated using the JGI annotation pipeline. The genome size, 21.1 MB, was similar to that of the biotechnological "workhorse", R. toruloides. Comparative analyses of the $R$. diobovata genome sequence with those of other Rhodotorula species, Yarrowia lipolytica, Phaffia rhodozyma, Lipomyces starkeyi, and Sporidiobolus salmonicolor, were conducted, with emphasis on the carotenoid and neutral lipid biosynthesis pathways. Amino acid sequence alignments of key enzymes in the lipid biosynthesis pathway revealed why the activity of malic enzyme and ATP-citrate lyase may be ambiguous in $Y$. lipolytica and L. starkeyi. Phylogenetic analysis showed a close relationship between $R$. diobovata and $R$. graminis WP1. Dot-plot analysis of the coding sequences of the genes crtYB and ME1 corroborated sequence homologies between sequences from $R$. diobovata and $R$. graminis. There was, however, nonsequential alignment between crtYB CDS sequences from $R$. diobovata and those from $X$. dendrorhous. This research presents the first genome analysis of $R$. diobovata with a focus on its biotechnological potential as a lipid and carotenoid producer.

Keywords: lipid biosynthesis; carotenoid biosynthesis; Rhodotorula diobovata; oleaginous yeasts; comparative genomics

\section{Introduction}

Oleaginous yeasts store more than $20 \%$ of their dry cell weight as triglycerides (TAGs). With a projected world population of 9.9 billion in 2050 [1], the course to cleaner and sustainable energy would require continuous research, development, and implementation of renewable energy strategies. The production of biofuels from oleaginous microorganisms has been widely researched as an alternative to traditional diesel from fossil fuels [2,3]. In addition to their application in biodiesel production, some oleaginous microorganisms have other biotechnological applications in nutritional and nutraceutical supplements, cosmetics, and medical and lipid research [4]. Some oleaginous yeasts are amenable to genetic engineering manipulations. In fact, Rhodotorula toruloides has been dubbed a "workhorse" for biotechnological applications [5]. While R. toruloides has been widely studied and engineered for various applications, other Rhodotorula species remain under characterized. One of those is the carotenogenic and oleaginous yeast, Rhodotorula diobovata.

Rhodotorula diobovata (Basionym, Rhodosporidium diobovatum) is an oleaginous, marine red yeast that can concomitantly synthesize quality single-cell oils for biodiesel production and health-promoting compounds, carotenoids, which are useful as food colorants in the 
food industry [6]. R. diobovata is capable of producing high biomass, with high cell-specific yields of triacylglycerides (TAGs) when grown on waste substrates such as biodieselderived waste glycerol [7]. This character makes this yeast even more attractive, because a reduction in the cost of the substrate will have a positive effect on the cost of biodiesel production. $R$. diobovata has been used in bioremediation, as it is able to assimilate high concentrations of nitrogen [8], and it has been found to thrive under varying culture conditions $[9,10]$. This study presents the first whole-genome sequence analysis and annotation of $R$. diobovata and compares the genome of $R$. diobovata with other oleaginous and carotenogenic yeasts with a focus on their lipid and carotenoid biosynthesis pathways.

\section{Materials and Methods}

\subsection{Microorganism and DNA Extraction}

Rhodotorula diobovata, UCDFST 08-225, was obtained from the Phaff Yeast Culture Collection, University of California, Davis (UCDFST). Cells were revived in YPD broth (20 g/L dextrose, $10 \mathrm{~g} / \mathrm{L}$ yeast extract, and $20 \mathrm{~g} / \mathrm{L}$ peptone) and incubated at $30^{\circ} \mathrm{C}$ for $48 \mathrm{~h}$ (h). The inoculum was streaked on YPD agar plates containing $20 \mathrm{~g} / \mathrm{L}$ dextrose, $10 \mathrm{~g} / \mathrm{L}$ yeast extract, $20 \mathrm{~g} / \mathrm{L}$ peptone, and $15 \mathrm{~g} / \mathrm{L}$ agar and incubated at $30{ }^{\circ} \mathrm{C}$ for $24 \mathrm{~h}$. Colonies from the agar plates were directly inoculated into YPD broth containing $10 \mathrm{~g} / \mathrm{L}$ yeast extract, $20 \mathrm{~g} / \mathrm{L}$ peptones, and $20 \mathrm{~g} / \mathrm{L}$ glucose and grown at $30^{\circ} \mathrm{C}$ and $150 \mathrm{rpm}$ for $24 \mathrm{~h}$.

To extract genomic DNA, $10 \mathrm{~mL}$ of culture was transferred into a falcon tube and centrifuged at $3000 \times g$ for $5 \mathrm{~min}$ at $4{ }^{\circ} \mathrm{C}$. Cell pellets were resuspended in phosphate buffer (PBS) and washed by centrifugation, followed by resuspension in PBS three times. A small aliquot $(500 \mu \mathrm{L})$ of cells was transferred to screw-capped Eppendorf for cell rupture. Cell rupture was carried out by bead-beating with about $20 \mathrm{mg}$ of $0.25 \mathrm{~mm}$ glass beads in a rotary bead-beater (Fisherbrand Bead Mill 24 Homogenizer) with three to five $30 \mathrm{~s}$ (s) cycles. To separate the debris from the lysate, tubes were centrifuged at $16,000 \times g$ for $20 \mathrm{~min}$ at $4{ }^{\circ} \mathrm{C}$. DNA extraction and purification were conducted using the Promega Wizard ${ }^{\circledR}$ Genomic DNA Purification Kit (Promega, Madison, WI, USA). DNA extraction was done in triplicate. Concentrations of the resulting sample were measured using Thermo Scientific's NanoDrop 1000 spectrophotometer (Thermo Fisher Scientific Inc., Madison, WI, USA).

\subsection{Genome Sequencing and Annotation}

The genome of the haploid strain, $R$. diobovata $08-225$, was sequenced using the Ion Torrent (IT) PGM and Illumina Miseq platforms. A total of four libraries were constructed, resulting in 3,051,898 reads from the IT platform, as well as 9,949,684 reads, 8,240,002 reads, and 5,899,760 reads from three Illumina libraries. The Illumina libraries had insert sizes of 300, 400, and $700 \mathrm{bp}$, while the IT library was $500 \mathrm{bp}$. The quality of the sequences was assessed using FastQC, and read error correction was carried out by Quake and Pollux error correction software. The Abyss Genome assembler resulted in the best hybrid assembly of Quake-corrected sequences from the Illumina platform and Pollux-corrected sequences from the IT platform [11,12].

To obtain a more reliably annotated genome, with information on exons, splicing sites, and alternative splicing, RNA-Seq was carried out on samples from mid-log and early stationary phase cells using the Illumina HiSeq platform. Transcripts were de novo assembled using rnaSPAdes and Trinity combined with two read-trimming tools: Trim Galore and Trimmomatic. Transrate was used to evaluate the quality of the assemblies. The rnaSPAdes assembler produced the best-quality assembly with samples from the mid-log phase giving a transrate score of 0.08 .

The assembled genome and transcriptome were submitted to the Joint Genome Institute's (JGI) MycoCosm platform and annotated using their annotation pipeline [13]. The annotated genome was further subjected to manual curation using Artemis [14]. This Whole-Genome Shotgun project has been deposited at DDBJ/ENA/GenBank under the accession SOZI00000000. The version described in this paper is version SOZI01000000. 


\subsection{Comparative Genomics and Phylogenetic Analyses}

Sequence datasets were created for proteins of interest, including malic enzyme, ATP-citrate lyase, acetyl-CoA acetyltransferase, bifunctional lycopene cyclase/phytoene synthase, and phytoene desaturase by BLASTP search. Phylogenetic analyses between these metabolic enzymes were used to infer sequence similarity among other oleaginous yeasts. Differences in the activity of these enzymes reported in the literature for some oleaginous yeasts $[15,16]$ informed the choice of oleaginous yeast sequences included in the phylogenetic analysis. Prior to multiple sequence alignments, redundant sequences were eliminated from the dataset using cd-hit (http://cd-hit.org). The sequences were then aligned by the MAFFT multiple sequence alignment program using the iterative refinement method FFT-NS-i [17]. Phylogenetic trees were constructed using protein maximum likelihood (PROML), and statistical support was obtained by a bootstrapping method 100 times. LAST was used to align DNA sequences in select scaffolds in R. graminis versus $R$. diobovata. All programs were accessed using the comprehensive bioinformatics system, BIRCH [18].

\section{Results and Discussion}

\subsection{General Features of the Rhodotorula Diobovata Genome}

Statistics of the genome assembly are shown in Table 1 (scaffold length distributions and genome coverage calculations are shown in Tables S1 and S2). De novo assembly of $R$. diobovata 08-225 produced 361 scaffolds, with the largest scaffold being $0.56 \mathrm{Mbp}$. The scaffold L50 number was $0.12 \mathrm{Mbp}$, the GC content was $66.9 \%$, and the size of the genome was $21.1 \mathrm{MB}$, which is similar to the genome size of other red oleaginous yeasts. R. graminis WP1 has a genome size of 21.01 MB [19], R. glutinis ATCC 204,091 has a size of 20.48 MB, R. toruloides NP11 is 20.22 MB [20], and Rhodotorula sp. JG-1b has a genome size of 19.39 MB [21]. The draft genome sequence of $R$. diobovata annotated by the JGI pipeline is estimated to have a total of 7970 protein-coding genes with an average of 5.95 exons per gene and an average protein length of 510 amino acids. While yeasts such as Saccharomyces cerevisiae generally have about 5800 coding genes with an average of 1 exon per gene, it is usual for Rhodotorula species to have a higher number of protein-coding genes and exons per gene. For example, 8171 protein-coding genes were predicted in $R$. toruloides NP11, with an average of 6.26 exons per gene [13].

Table 1. Summary of genome assembly statistics.

\begin{tabular}{ll}
\hline Genome assembly size $(\mathrm{Mbp})$ & 21.14 \\
\hline Genome coverage & $44 \times$ \\
\hline Number of contigs & 614 \\
\hline Number of scaffolds & 361 \\
\hline Number of scaffolds $\geq 2 \mathrm{Kbp}$ & 336 \\
\hline Scaffold N50 & 55 \\
\hline Scaffold L50 (Mbp) & 0.12 \\
\hline Number of gaps & 253 \\
\hline Percentage of scaffold length in gaps & $0.10 \%$ \\
\hline Three largest scaffolds $(\mathrm{Mbp})$ & $0.56,0.49,0.41$ \\
\hline
\end{tabular}

\subsection{Analysis of Proteins Associated with De Novo Fatty Acid Biosynthesis}

The biosynthesis of fatty acids in oleaginous yeasts may be either de novo or ex novo $[22,23]$. This study focuses on the de novo biosynthesis pathway, which involves the fermentation of sugars and related substrates. Biochemically, the "oleaginicity" of a microorganism is defined by the presence of certain key enzymes: isocitrate dehydrogenase, adenosine triphosphate (ATP)-citrate lyase, malic enzyme, and fatty acid synthase [24]. 
ATP-citrate lyase is responsible for providing acetyl subunits for fatty acid biosynthesis. Malic enzyme is involved in the generation of nicotinamide adenine dinucleotide phosphate (NADPH) to reduce acetyl subunits, which form the backbone of fatty acids [25]. Here, ATPcitrate lyase and malic enzyme from $R$. diobovata are analyzed, and datasets for sequence alignment were created to include sequences from other oleaginous yeasts.

\subsection{Malic Enzyme}

Malic enzyme (ME) catalyzes the oxidative decarboxylation of malate to pyruvate, producing NADPH, which is necessary for fatty acid biosynthesis. Ratledge [26] reviewed the role of ME as a producer of NADPH in oleaginous microorganisms and concluded that ME could not be the only source of NADPH in oleaginous microorganisms, because some oleaginous microorganisms, such as Y. lipolytica and L. starkeyi, were found to possess just one malic enzyme present in the mitochondria [27]. Fatty acid biosynthesis is a cytosolic process, and the absence of a cytosolic malic enzyme in these yeasts suggests that the supply of NADPH for fatty acid biosynthesis may be from alternative pathways, such as the pentose phosphate pathway.

R. diobovata, like many other Rhodotorula species, has 2 MEs (DMC30DRAFT_420609 and DMC30DRAFT_357466) with predicted lengths of 633 and 613 amino acids, respectively. This is similar to the number of proteins annotated as ME in the oleaginous yeast Papiliotrema laurentii [16] but dissimilar to the ten genes annotated as encoding ME in Trichosporon fermentans [28]. Phylogenetic analyses based on the MAFFT alignment of amino acid sequences of malic enzyme revealed that MEs from $R$. diobovata (TNY17208 and TNY24152) were most closely related with those from R. graminis WP1 (KPV73817 and KPV76802) (Figure 1).

A WebLogo plot of the aligned sequences (Figure S1) was constructed to identify the regions where specific amino acids were conserved before a more in-depth analysis of the alignment was carried out. In addition to the divalent ion-binding sites (Figure S2), alignment analysis revealed two dinucleotide-binding sites associated with NAD and NADP. These conserved regions (highlighted in Figure 2) have glycine-rich sequences [29,30]. Sequence similarities within the putative mitochondrial putative cytosolic enzymes are obvious in the NAD-linked and NADP-linked regions. The putative cytosolic enzymes have aliphatic and nonpolar leucine (L) at Position 216 instead of the acidic and polar glutamine (Q) observed in the putative mitochondrial enzymes at the NAD-linked region. Position 369 of the alignment shows all of the putative cytosolic enzymes contained hydroxylic and polar serine (S) instead of aliphatic and nonpolar alanine (A) observed in the mitochodrial enzymes. For the NADP-linked region, Y. lipolytica, L. starkeyi, and X. dendrorhous have a hydroxyl or sulfur-containing amino acid at Position 371 of the alignment rather than the aliphatic or aromatic amino acids (valine, leucine, and tyrosine) observed in the same position for puciniomycotas examined in this analysis. 


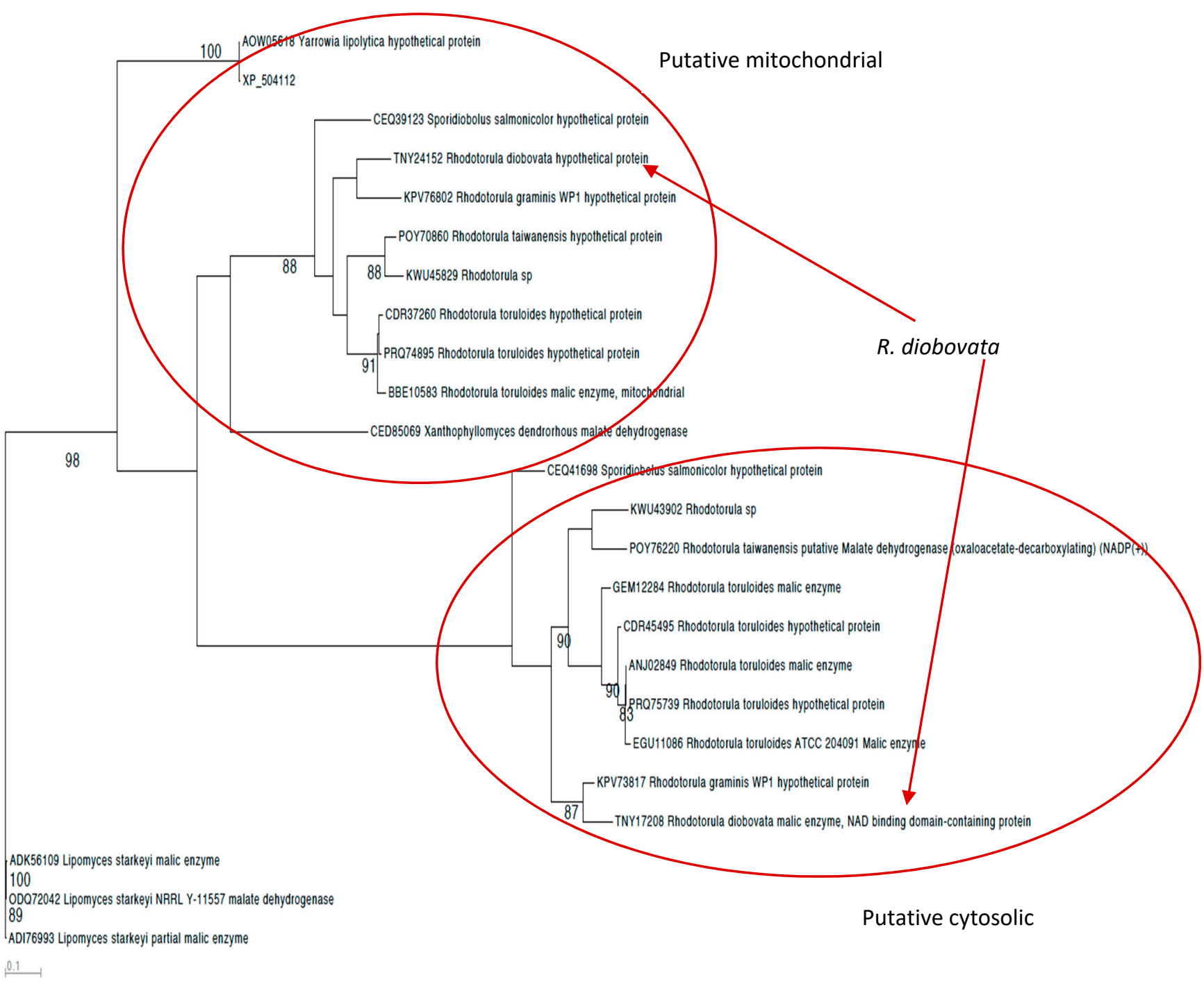

Figure 1. PROML phylogenetic tree constructed from an alignment of malic enzyme sequences from Rhodotorula species, Yarrowia, Lipomyces, and Xanthophyllomyces. Rhodotorula diobovata sequences are pointed out, showing cytosolic and mitochondrial positioning. The significant nodes on the tree with bootstrap support values $>70 \%$ are highlighted.

While $X$. dendrorhous is not established as an oleaginous yeast, Y. lipolytica and L. starkeyi are established oleaginous yeasts, the ME activities of which have been unclear, as they do not possess cytosolic ME. In fact, the sequence in the NADP-ME-conserved regions has been associated with $Y$. lipolytica's preference of $\mathrm{NAD}^{+}$over $\mathrm{NADP}^{+}$as a cofactor [15]. Zhang et al. [15] found that $\mathrm{NADP}^{+}$-dependent activity was only $1 \%$ of $\mathrm{NAD}^{+}$-dependent activity and that $\mathrm{NAD}^{+}$had a much lower $\mathrm{Km}$ value. Another alternative for NADPH supply in Y. lipolytica and L. starkeyi is the cytosolic $\mathrm{NADP}^{+}$-dependent isocitrate dehydrogenase [26]. 


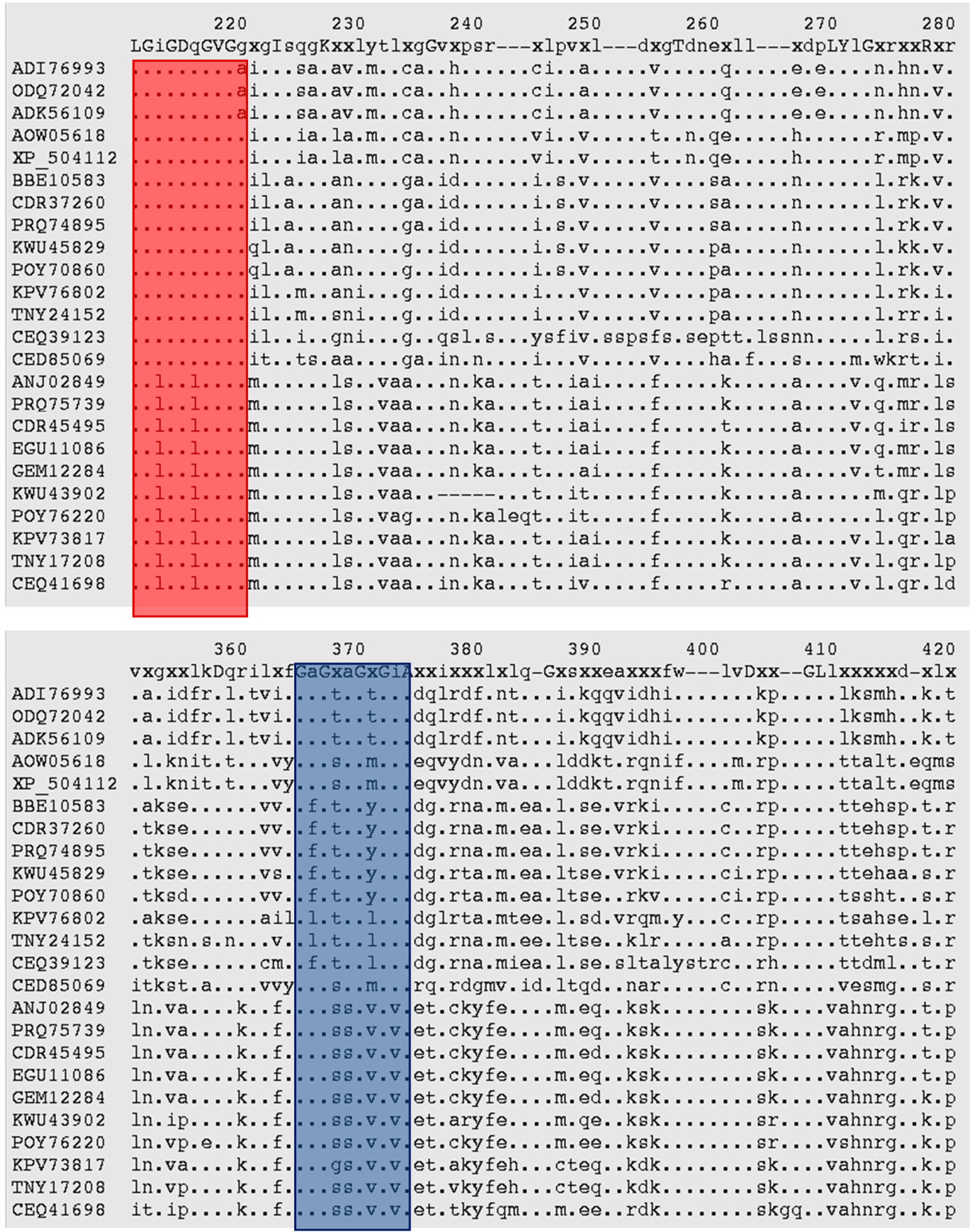

Figure 2. MAFFT alignment of malic enzymes (MEs) showing dinucleotide-binding sites. The red box is for NAD-linked MEs, and the blue box is for NADP-linked MEs [29]. Identical amino acid residues in all the enzymes are signified by a dot (.) ADI76993, Lipomyces starkeyi; ODQ72042, Lipomyces starkeyi NRRL Y-11557; ADK56109, Lipomyces starkeyi; AOW05618, Yarrowia lipolytica CLIB89(W29); XP_504112, Yarrowia lipolytica CLIB122; BBE10583, Rhodotorula toruloides NBRC10032; CDR37260, Rhodotorula toruloides CECT1137; PRQ74895, Rhodotorula toruloides NBRC0880; KWU45829, Rhodotorula sp. JG-1b; POY70860, Rhodotorula taiwanensis; KPV76802, Rhodotorula graminis WP1; TNY24152, Rhodotorula diobovata; CEQ39123, Sporidiobolus salmonicolor; CED85069, Xanthophyllomyces dendrorhous; ANJ02849, Rhodotorula toruloides IFO0880; PRQ75739, Rhodotorula toruloides NBRC0880; CDR45495, Rhodotorula toruloides CECT1137; EGU11086, Rhodotorula toruloides ATCC 204091; GEM12284, Rhodotorula toruloides NBRC10032; KWU43902, Rhodotorula sp. JG-1b; POY76220, Rhodotorula taiwanensis; KPV73817, Rhodotorula graminis WP1; TNY17208, Rhodotorula diobovata; CEQ41698, Sporidiobolus salmonicolor. 


\subsection{ATP-Citrate Lyase}

ATP-citrate lyase (ACL), also annotated as ATP-citrate synthase, is an enzyme characteristic of oleaginous microorganisms. While the presence of ACL does not confer oleaginicity, oleaginous microorganisms have been found to possess it [31]. ACL cleaves citrate in the cytosol to produce acetyl-CoA and oxaloacetate. The alignment of $R$. diobovata ACL amino acid sequences with those from other oleaginous yeasts shows a close relationship between $R$. diobovata and $R$. toruloides NBRC10032 (Figure S3). This analysis shows that Rhodotorula species appear to have only one gene encoding the ACL enzyme, unlike the oleaginous Saccharomycotina species, which possess two genes encoding two ACL subunits, while eight (8) genes encoded for ACL in T. fermentans, six (6) in A. oryzae, and seven (7) in R. opacus genomes [28].

The protein ATP-citrate synthase in $R$. diobovata has 1124 amino acids, with 3 conserved regions: succinyl-CoA synthetase (SucC) beta subunit (cl33750). ATP-citrate lyase citratebinding (Citrate_bind superfamily) (cl29684), and ATP-citrate (pro-S)-lyase (PLN02522) (cl31895) (Figure 3). Other Rhodotorula species analyzed only contained the cl31895 and cl29684 conserved regions on one ACL (Figure 3). SucC is, however, a conserved domain containing the citrate-binding sites cl29684 and cl33750, which are, therefore, overlapping homologous superfamilies [32]. Oleaginous Saccharomycotina, which includes Y. lipolytica and L. starkeyi, have conserved domains cl29684 and cl31895 on separate subunits. This may explain the difference in ACL expressions under lipogenic conditions. While ACL gene expressions did not correlate with the lipid accumulation phase in Y. lipolytica [33], expression levels of ACL in R. toruloides were high in the lipid accumulation phase [20].

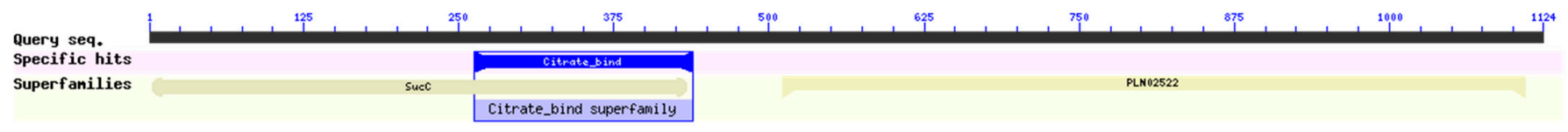

Figure 3. Graphical summary of conserved domains on ATP-citrate synthase in Rhodotorula diobovata obtained from the Conserved Domain Database [34].

\subsection{Analysis of Proteins Associated with Carotenoid Biosynthesis}

Carotenoid biosynthesis is divided into three steps: the synthesis of mevalonate, followed by the synthesis of isopentenyl pyrophosphate (IPP), which leads to the biosynthesis of terpenoids [35]. The first step in the biosynthesis of mevalonate in red oleaginous yeasts is the formation of acetoacetyl-CoA from acetyl-CoA in the cytosol. This is achieved by the action of a thiolase, acetyl-CoA C-acetyltransferase (ACAT), which catalyzes the transfer of an acetyl group to acetyl-CoA. The addition of another acetyl group catalyzed by 3-hydroxy-3-methylglutaryl-CoA (HMG-CoA) synthase is the second step in mevalonate biosynthesis. Another acetyl group is then added to 3-hydroxy-3-methylglutaryl-CoA synthesized in the second step. This reaction is catalyzed by HMG-CoA reductase, and it is considered the rate-limiting step in carotenoid biosynthesis [36]. IPP is synthesized by a series of phosphorylation reactions that lead to the final stage of terpenoid biosynthesis (Figure 4).

Rhodotorula species typically synthesize $\beta$-carotene, torulene, and torularhodin $[37,38]$. However, $R$. diobovata favors the biosynthesis of torulene and torularhodin over $\beta$-carotene under varying growth conditions [6]. In addition to the comparative analysis of thiolase, the first enzyme in the biosynthetic pathway of carotenoids, an assessment of bifunctional lycopene cyclase/phytoene synthase and phytoene desaturase was carried out. 


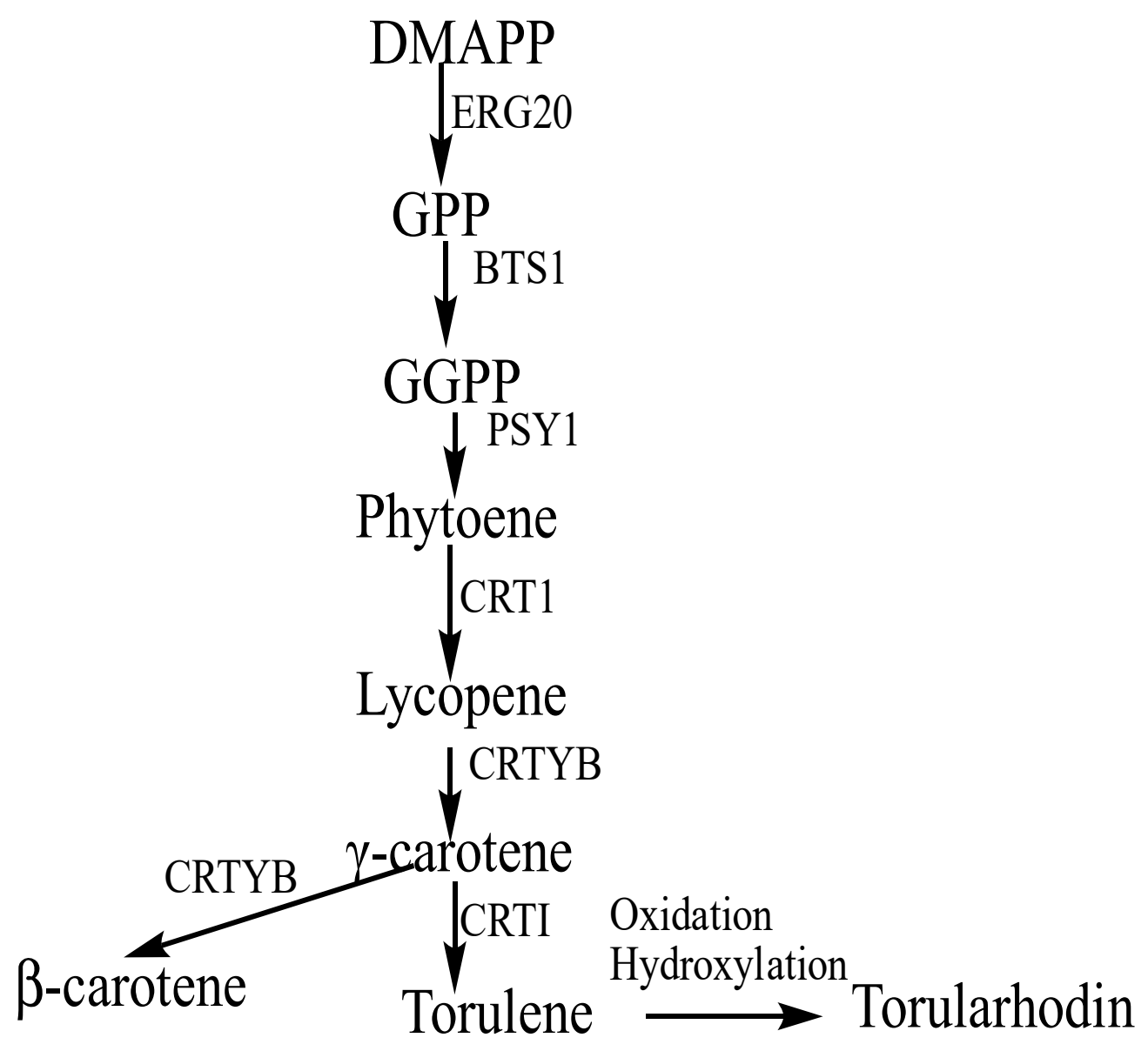

Figure 4. Isoprenoid biosynthesis pathways. DMAPP, dimethylallyl pyrophosphate; ERG20, farnesyl pyrophosphate synthetase; GPP, geranyl pyrophosphate; BTS1(CRTE), geranylgeranyl pyrophosphate synthase; GGPP, geranylgeranyl pyrophosphate; PSY1(CRTB), phytoene synthase; CRTI, phytoene desaturase; CRTYB (CRTY), bifunctional lycopene cyclase/phytoene synthase.

\subsection{Acetyl-CoA C-Acetyltransferase}

Acetyl-CoA C-acetyltransferase (encoded by ERG10) belongs to the ubiquitous thiolase family, and it catalyzes the reaction from acetyl-CoA to acetoacetyl-CoA. There are two broad types of thiolase-degradative thiolases (3-ketoacyl-CoA thiolase) and biosynthetic thiolases (acetoacetyl-CoA thiolase), which are involved in the first step of mevalonate synthesis [39]. While there are five (5) distinct genes encoding thiolase enzymes in the R. diobovata (Accession Numbers: TNY24221, TNY23669, TNY19355, TNY19167, TNY16984), TNY19355 revealed the best alignment with the thiolase-conserved domain, cd00751, with an E-value of 0 .

Alignment analysis of acetyl-CoA C-acetyltransferase from R. diobovata, P. rhodozyma, $R$. toruloides, S. salmonicolor, R. graminis, and R. taiwanensis shows the two conserved cysteine residues at the $\mathrm{N}$-terminus and $\mathrm{C}$-terminus involved in the acyl-enzyme intermediate formation and active site base, respectively [40]. Phylogenetic analyses revealed a close relationship between TNY19355 (R. diobovata) and KPV74338 (R. graminis WP1) (Figure S4).

\subsection{Bifunctional Lycopene Cyclase/Phytoene Synthase}

Bifunctional lycopene cyclase/phytoene synthase is responsible for phytoene synthesis, the cyclization of lycopene to $\gamma$-carotene, and further cyclization to $\beta$-carotene (Figure 4). The gene coding for this bifunctional enzyme in $R$. diobovata crtYB was studied by Guo et al. [41]. The protein contains two copies of the CarR_dom_SF domain, which is the lycopene cyclase domain, on the N-terminus. The Isoprenoid_Biosyn_C1 (phytoene synthase) superfamily domain is located at the C-terminal (Figure 5). Phylogenetic anal- 
yses of the 594 amino acid sequence of crtYB from $R$. diobovata (AGT42003) with those from other Rhodotorula species, P. rhodozyma, S. salmonicolor, and S. pararoseus, revealed that the $R$. diobovata crtYB was most closely related to the crtYB of R. graminis WP1. An increase in the expression of this gene has been linked to increased carotenoid production in R. toruloides under light conditions [42], and it has been shown to be transcriptionally regulated in $X$. dendrorhous [43]. This gene is also one of the most favored in the engineering of noncarotenogenic yeast strains for the production of $\beta$-carotene [44].

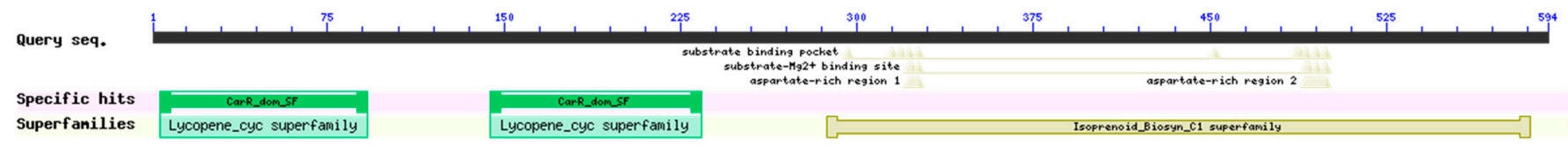

Figure 5. Graphical summary of putative conserved domains of the bifunctional lycopene cyclase/phytoene synthase of Rhodotorula diobovata obtained from the Conserved Domain Database [34].

\subsection{Phytoene Desaturase}

Phytoene desaturation is a rate-limiting step in the carotenoid biosynthetic pathway [45]. Phytoene desaturase (phytoene dehydrogenase), encoded by the crtI gene, catalyzes the formation of lycopene from phytoene. The same enzyme also catalyzes the formation of torulene from $\gamma$-carotene (Figure 4). Phytoene desaturase (PDS), TNY20974 (551 aa) from $R$. diobovata UCDFST 08-225 with a conserved region, crtI_fam, spanning Positions 7 to 505, was aligned with the enzyme from other Basidiomycota species, and this alignment revealed a $99 \%$ amino acid (aa) sequence identity with a previously characterized PDS from $R$. diobovatum ATCC 2527. Guo et al. [46] found that this crtI gene encodes a 547 amino acid PDS protein with a molecular mass of $60.45 \mathrm{kDa}$ and has 7 introns. Its amino acid sequence (AHB14354) was shown to have a $46 \%$ aa sequence identity with the X. dendrorhous PDS enzyme and a $45 \%$ aa sequence identity with the PDS of Blakeslea trispora, while TNY20974 from this work showed a $47 \%$ aa sequence identity with the PDS of $X$. dendrorhous. Phylogenetic analysis showed a close relationship between the $R$. diobovata PDS amino acid sequences and the PDS from $R$. graminis WP1.

From the sequence analysis of enzymes in the lipid and carotenoid biosynthesis pathways, there is a general close alignment between R. graminis WP1 and R. diobovata. To further examine the sequence similarities observed between $R$. graminis WP1 and $R$. diobovata, dot-plot analysis of the coding sequences (CDS) of crtYB and ME genes from these yeasts was constructed. CDS of ME and crtYB from Y. lipolytica and X. dendrorhous, respectively, were also included in this analysis. The dot-plot was used to visually inspect sequences, and it revealed similarities and some frameshifts (Figures 6 and 7). Cytosolic and mitochondrial ME sequences of $R$. diobovata and $R$. graminis show sequence homology evident from the continuous match. There is, however, an insertion observed between the two cytosolic sequences (Figure 6a) [47].

Cytosolic and mitochondrial ME sequences of $R$. diobovata were also plotted against CDS of ME from Y. lipolytica (Figure $6 \mathrm{c}, \mathrm{d}$ ), which is localized in the mitochondria. The organizational difference observed here may be responsible for how malic enzyme operates in Y. lipolytica compared to other oleaginous yeasts. Dot-plots of CDS sequences of crtYB of $R$. diobovata versus $R$. graminis also showed a continuous match between the two sequences (Figure 7a). Sequences from $R$. diobovata versus $X$. dendrorhous, however, appear to have a series of sequence deletion events (Figure $7 \mathrm{~b}$ ). The study of these types of insertions, deletions, and rearrangements is important, as they may alter phenotypes. Despite the phenotypes of oleaginous and carotenogenic yeasts being similar, genome and sequencestructure data can provide useful information needed prior to gene editing. This then forms a basis for genetic engineering for biotechnological applications. 

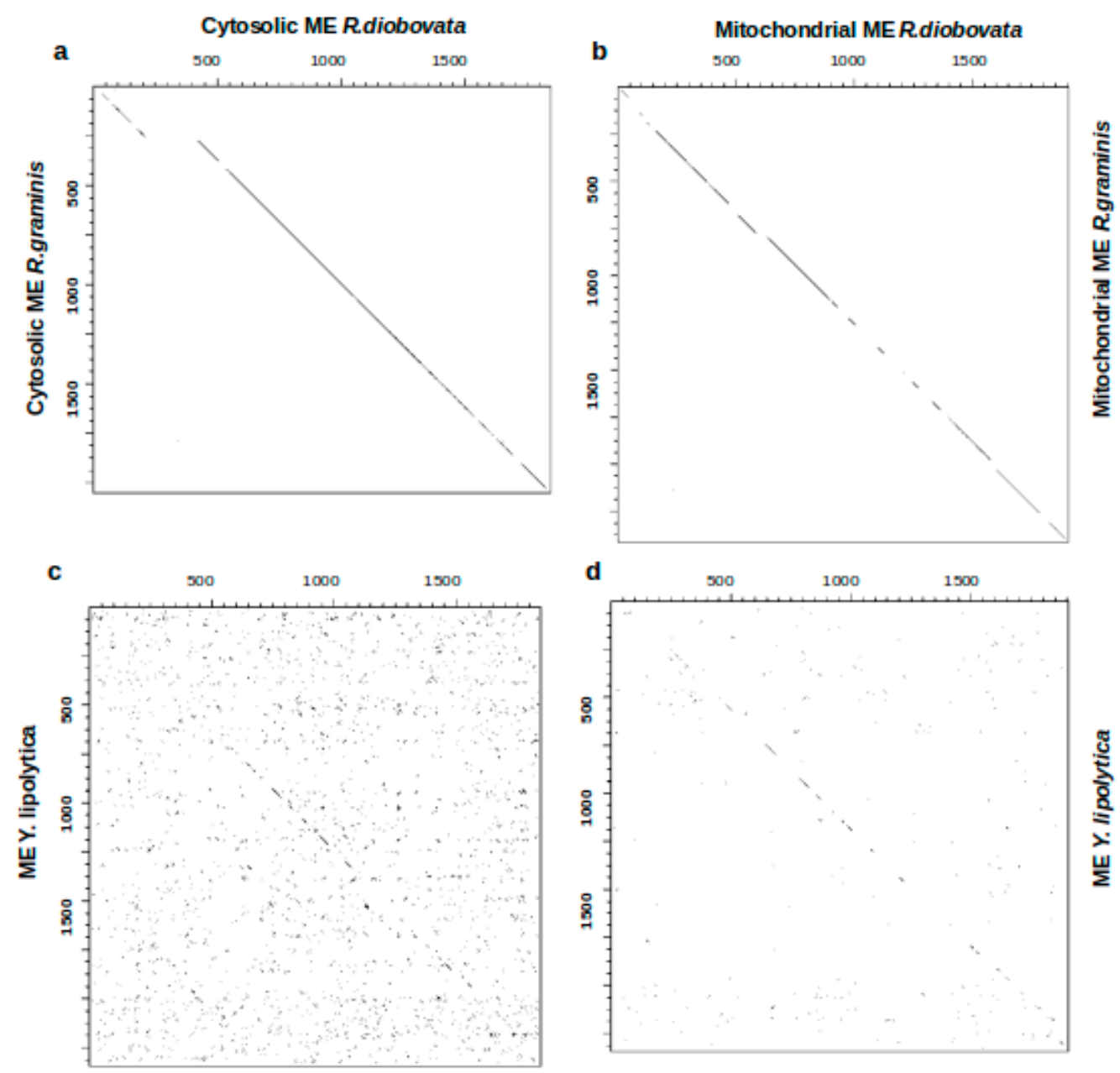

Figure 6. Dot-plots of individual protein-coding sequences constructed by dotter showing mostly a continuous match between sequences. (a) Cytosolic ME in Rhodotorula diobovata on the $x$-axis and cytosolic ME in Rhodotorula graminis on the $y$-axis; (b) mitochondrial ME in R. diobovata on the $x$-axis and mitochondrial ME in R. graminis on the $y$-axis; (c) cytosolic ME in $R$. diobovata on the $x$-axis and ME in Yarrowia lipolytica on the $y$-axis; (d) mitochondrial ME in $R$. diobovata on the $x$-axis and Y. lipolytica ME in on the $y$-axis.
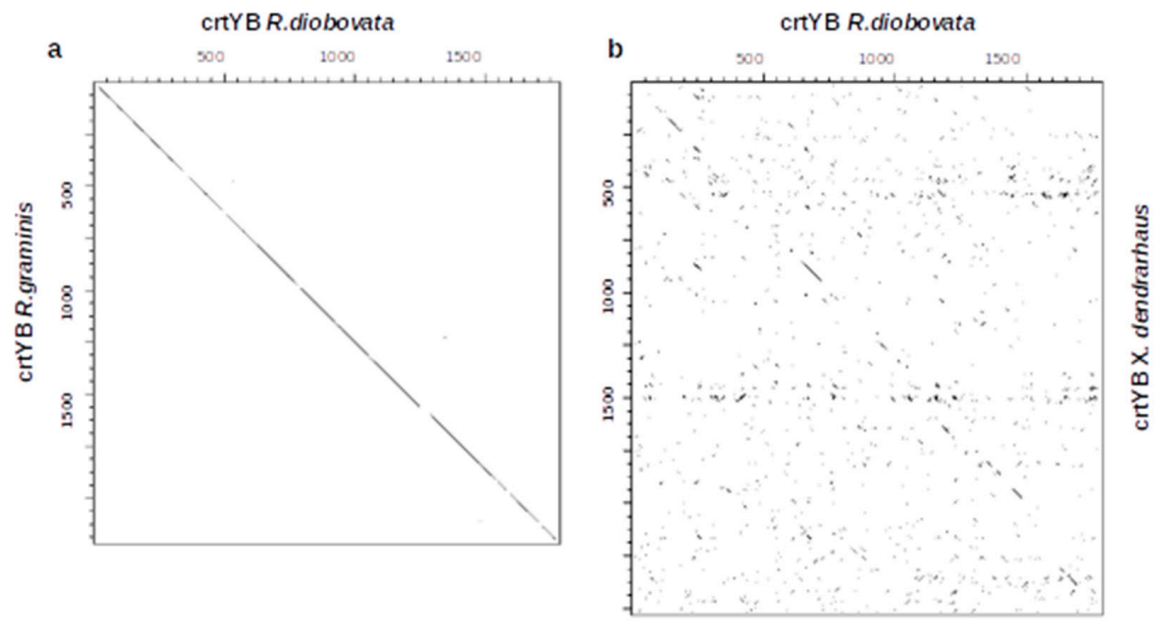

Figure 7. Dot-plots of individual protein-coding sequences constructed by dotter showing (a) a continuous sequence match of crtYB in Rhodotorula diobovata on the $x$-axis and crtYB sequence from Rhodotorula graminis on the $y$-axis and (b) regions that are strongly conserved between crtYB of $R$. diobovata and the crtYB sequence from Xanthophyllomyces dendrorhous, with little evident conservation in the rest of the lengths of the two protein-coding sequences. 


\section{Conclusions}

This study presented the whole-genome sequencing of an oleaginous and carotenogenic basidiomycete, Rhodotorula diobovata, and revealed core differences between the amino acid sequences of $R$. diobovata proteins involved in the lipid and carotenoid biosynthesis pathways and those from other oleaginous yeasts. The unconventional mode of action of malic enzyme and ATP-citrate lyase in L. starkeyi and Y. lipolytica may be connected to their sequence organization. Dot-plot analysis of the coding sequences of malic enzyme and crtYB confirmed sequence homologies between $R$. diobovata and $R$. graminis sequences. There was, however, nonsequential alignment between crtYB CDS sequences from $R$. diobo$v a t a$ and those from $X$. dendrorhous. This research presents useful information for genetic engineering and the potential exploration of $R$. diobovata as a biotechnological "workhorse".

Supplementary Materials: The following are available online at https:/ /www.mdpi.com/article/ 10.3390/jof7040320/s1, Figure S1: Weblogo plot of aligned sequences from Rhodotorula diobovata, Lipomyces starkeyi, Rhodotorula toruloides NP11, Rhodotorula graminis WP1, Rhodotorula sp. JG-1b, Sporidiobolus salmonicolor, Phaffia rhodozyma, Yarrowia lipolytica, and Rhodotorula taiwanensis. Plot shows relative frequency of each amino acid at each position. Red highlighted boxes show the dinucleotide binding sites while the blue boxes show the divalent ion binding sites. Figure S2. MAFFT alignment of malic enzymes showing the divalent ion binding sites [Borsch and Westhoff, 1990]. Identical amino acid residues in all the enzymes are signified by a dot (.) ADI76993, Lipomyces starkeyi; ODQ72042, Lipomyces starkeyi NRRL Y-11557; ADK56109, Lipomyces starkeyi; AOW05618, Yarrowia lipolytica CLIB89(W29); XP_504112, Yarrowia lipolytica CLIB122; BBE10583, Rhodotorula toruloides NBRC10032; CDR37260, Rhodotorula toruloides CECT1137; PRQ74895, Rhodotorula toruloides NBRC0880; KWU45829, Rhodotorula sp. JG-1b; POY70860, Rhodotorula taiwanensis; KPV76802, Rhodotorula graminis WP1; TNY24152, Rhodotorula diobovata; CEQ39123, Sporidiobolus salmonicolor; CED85069, Xanthophyllomyces dendrorhous; ANJ02849, Rhodotorula toruloides IFO0880; PRQ75739, Rhodotorula toruloides NBRC0880; CDR45495, Rhodotorula toruloides CECT1137; EGU11086, Rhodotorula toruloides ATCC 204091; GEM12284, Rhodotorula toruloides NBRC10032; KWU43902, Rhodotorula sp. JG1b; POY76220, Rhodotorula taiwwanensis; KPV73817, Rhodotorula graminis WP1; TNY17208, Rhodotorula diobovata; CEQ41698, Sporidiobolus salmonicolor. Figure S3. PROML phylogenetic tree constructed from an alignment of ATP: Citrate lyase sequences from Rhodotorula sp., Yarrowia, Lipomyes, and Xanthophyllomyces. Analysis shows the relationship between the two ACL subunits of Y. lipolytica and L. starkeyi. The significant nodes on the tree with bootstrap support values $>70 \%$ are highlighted. Figure S4. PROML phylogenetic tree constructed from a MAFFT alignment of Acetyl-CoA C-acetyltransferase sequences from Rhodotorula species, Yarrowia, Lipomyes, Sporidobolus and Xanthophyllomyces. The significant nodes on the tree with bootstrap support values $>70 \%$ are highlighted. Figure S5. Scaffold length distribution of all 361 scaffolds from the De novo assembly of $R$. diobovata 08-225. Table S1. Scaffold length distribution for the 361 scaffolds used to assemble the Rhodotorula diobovata genome Table S2. Genome coverage calculation for Illumina and Ion Torrent reads.

Author Contributions: Conceptualization, methodology, and writing-original draft preparation, I.F.; data curation, software, and formal analysis, B.F.; supervision, writing - reviewing and editing, funding acquisition, and resources, D.B.L. All authors have read and agreed to the published version of the manuscript.

Funding: This research was supported by a Natural Sciences and Engineering Research Council of Canada (NSERC) Discovery grant (RGPIN-04945-2017) held by David B. Levin.

Institutional Review Board Statement: Not applicable.

Informed Consent Statement: Not applicable.

Data Availability Statement: This Whole-Genome Shotgun project has been deposited at DDBJ/ ENA/GenBank under the accession SOZI00000000. https://www.ncbi.nlm.nih.gov/bioproject/ ?term=rhodotorula\%20diobovata (accessed on 19 June 2019). 
Acknowledgments: The authors would like to thank the University of Manitoba NGS Sequencing Platform, McGill University and Génome Québec Innovation Centre, and Joint Genome Institute (JGI) for their technical assistance.

Conflicts of Interest: No conflict of interest declared.

\section{References}

1. Kaneda, T.; Greenbaum, C.; Kline, K. Population Reference Bureau. 2020 World Population Data Sheet Shows Older Populations Growing, Total Fertility Rates Declining. 2020. Available online: https://www.prb.org/2020-world-population-data-sheet (accessed on 30 November 2020).

2. Donot, F.; Fontana, A.; Baccou, J.C.; Strub, C.; Schorr-Galindo, S. Single Cell Oils (SCOs) from Oleaginous Yeasts and Moulds: Production and Genetics. Biomass Bioenergy 2014, 68, 135-150. [CrossRef]

3. Ochsenreither, K.; Glück, C.; Stressler, T.; Fischer, L.; Syldatk, C. Production Strategies and Applications of Microbial Single Cell Oils. Front. Microbiol. 2016, 7, 1539. [CrossRef]

4. Yurkov, A.M.; Vustin, M.M.; Tyaglov, B.V.; Maksimova, I.A.; Sineokiy, S.P. Pigmented Basidiomycetous Yeasts Are a Promising Source of Carotenoids and Ubiquinone Q10. Microbiology 2008, 77, 1-6. [CrossRef]

5. Park, Y.-K.; Nicaud, J.-M.; Ledesma-Amaro, R. The Engineering Potential of Rhodosporidium Toruloides as a Workhorse for Biotechnological Applications. Trends Biotechnol. 2018, 36, 304-317.

6. Nasirian, N.; Mirzaie, M.; Cicek, N.; Levin, D.B. Lipid and Carotenoid Synthesis by Rhodosporidium Diobovatum, Grown on Glucose versus Glycerol, and Its Biodiesel Properties. Can. J. Microbiol. 2018, 64, 277-289. [CrossRef]

7. Munch, G.; Sestric, R.; Sparling, R.; Levin, D.B.; Cicek, N. Lipid Production in the Under-Characterized Oleaginous Yeasts, Rhodosporidium babjevae and Rhodosporidium diobovatum, from Biodiesel-Derived Waste Glycerol. Bioresour. Technol. 2015, 185, 49-55. [CrossRef]

8. Civiero, E.; Pintus, M.; Ruggeri, C.; Tamburini, E.; Sollai, F.; Sanjust, E.; Zucca, P. Physiological and Phylogenetic Characterization of Rhodotorula Diobovata DSBCA06, a Nitrophilous Yeast. Biology 2018, 7, 39. [CrossRef]

9. Newell, S.Y.; Hunter, I.L. Rhodosporidium diobovatum sp. n., the Perfect Form of an Asporogenous Yeast (Rhodotorula Sp.)1. J. Bacteriol. 1970, 104, 503-508. [CrossRef]

10. Fakankun, I.; Mirzaei, M.; Levin, D.B. Impact of Culture Conditions on Neutral Lipid Production by Oleaginous Yeast. Methods Mol. Biol. 2019, 1995, 311-325.

11. Kelley, D.R.; Schatz, M.C.; Salzberg, S.L. Quake: Quality-Aware Detection and Correction of Sequencing Errors. Genome Biol. 2010, 11, R116. [CrossRef]

12. Marinier, E.; Brown, D.G.; McConkey, B.J. Pollux: Platform Independent Error Correction of Single and Mixed Genomes. BMC Bioinform. 2015, 16, 10. [CrossRef]

13. Nordberg, H.; Cantor, M.; Dusheyko, S.; Hua, S.; Poliakov, A.; Shabalov, I.; Smirnova, T.; Grigoriev, I.V.; Dubchak, I. The Genome Portal of the Department of Energy Joint Genome Institute: 2014 Updates. Nucleic Acids Res. 2014, 42, D26-D31. [CrossRef] [PubMed]

14. Carver, T.; Harris, S.R.; Berriman, M.; Parkhill, J.; McQuillan, J.A. Artemis: An Integrated Platform for Visualization and Analysis of High-Throughput Sequence-Based Experimental Data. Bioinformatics 2012, 28, 464-469. [CrossRef] [PubMed]

15. Zhang, H.; Zhang, L.; Chen, H.; Chen, Y.Q.; Ratledge, C.; Song, Y.; Chen, W. Regulatory Properties of Malic Enzyme in the Oleaginous Yeast, Yarrowia Lipolytica, and Its Non-Involvement in Lipid Accumulation. Biotechnol. Lett. 2013, 35, $2091-2098$. [CrossRef] [PubMed]

16. Moreira Vieira, N.; Zandonade Ventorim, R.; de Moura Ferreira, M.A.; Barcelos de Souza, G.; Menezes de Almeida, E.L.; Pereira Vidigal, P.M.; Nunes Nesi, A.; Gomes Fietto, L.; Batista da Silveira, W. Insights into Oleaginous Phenotype of the Yeast Papiliotrema laurentii. Fungal Genet. Biol. 2020, 144, 103456. [CrossRef] [PubMed]

17. Katoh, K.; Standley, D.M. MAFFT Multiple Sequence Alignment Software Version 7: Improvements in Performance and Usability. Mol. Biol. Evol. 2013, 30, 772-780. [CrossRef]

18. Fristensky, B. BIRCH: A User-Oriented, Locally-Customizable, Bioinformatics System. BMC Bioinf. 2007, 8, 54. [CrossRef] [PubMed]

19. Firrincieli, A.; Otillar, R.; Salamov, A.; Schmutz, J.; Khan, Z.; Redman, R.S.; Fleck, N.D.; Lindquist, E.; Grigoriev, I.V.; Doty, S.L. Genome Sequence of the Plant Growth Promoting Endophytic Yeast Rhodotorula Graminis WP1. Front. Microbiol. $2015,6,978$. [CrossRef]

20. Zhu, Z.; Zhang, S.; Liu, H.; Shen, H.; Lin, X.; Yang, F.; Zhou, Y.J.; Jin, G.; Ye, M.; Zou, H.; et al. A Multi-Omic Map of the Lipid-Producing Yeast Rhodosporidium Toruloides. Nat. Commun. 2012, 3, 1112. [CrossRef] [PubMed]

21. Goordial, J.; Raymond-Bouchard, I.; Riley, R.; Ronholm, J.; Shapiro, N.; Woyke, T.; LaButti, K.M.; Tice, H.; Amirebrahimi, M.; Grigoriev, I.V.; et al. Improved High-Quality Draft Genome Sequence of the Eurypsychrophile rhodotorula sp. JG1b, Isolated from Permafrost in the Hyperarid Upper-Elevation McMurdo Dry Valleys, Antarctica. Genome Announc. 2016, 4. [CrossRef]

22. Papanikolaou, S.; Aggelis, G. Lipids of Oleaginous Yeasts. Part II: Technology and Potential Applications. Eur. J. Lipid Sci. Technol. 2011, 113, 1052-1073. [CrossRef]

23. Papanikolaou, S.; Aggelis, G. Lipids of Oleaginous Yeasts. Part I: Biochemistry of Single Cell Oil Production. Eur. J. Lipid Sci. Technol. 2011, 113, 1031-1051. [CrossRef] 
24. Ratledge, C. Microbial Lipids: Commercial Realities or Academic Curiosities. In Industrial Applications of Single Cell Oils; AOCS Publishing: Boulder Urbana, IL, USA, 1992.

25. Akpinar-Bayizit, A. Fungal Lipids: The Biochemistry of Lipid Accumulation. Int. J. Chem. Eng. Appl. 2014, 5, 409-414. [CrossRef]

26. Ratledge, C. The Role of Malic Enzyme as the Provider of NADPH in Oleaginous Microorganisms: A Reappraisal and Unsolved Problems. Biotechnol. Lett. 2014, 36, 1557-1568. [CrossRef]

27. Morin, N.; Cescut, J.; Beopoulos, A.; Lelandais, G.; Le Berre, V.; Uribelarrea, J.-L.; Molina-Jouve, C.; Nicaud, J.-M. Transcriptomic Analyses during the Transition from Biomass Production to Lipid Accumulation in the Oleaginous Yeast Yarrowia lipolytica. PLoS ONE 2011, 6, e27966. [CrossRef] [PubMed]

28. Shen, Q.; Chen, Y.; Jin, D.; Lin, H.; Wang, Q.; Zhao, Y.-H. Comparative Genome Analysis of the Oleaginous Yeast Trichosporon Fermentans Reveals Its Potential Applications in Lipid Accumulation. Microbiol. Res. 2016, 192, 203-210. [CrossRef]

29. Börsch, D.; Westhoff, P. Primary Structure of NADP-Dependent Malic Enzyme in the Dicotyledonous C4 Plant Flaveria trinervia. FEBS Lett. 1990, 273, 111-115. [CrossRef]

30. Zhang, Y.; Adams, I.P.; Ratledge, C. Malic Enzyme: The Controlling Activity for Lipid Production? Overexpression of Malic Enzyme in Mucor circinelloides Leads to a 2.5-Fold Increase in Lipid Accumulation. Microbiology 2007, 153, 2013-2025. [CrossRef]

31. Ratledge, C. Regulation of Lipid Accumulation in Oleaginous Micro-Organisms. Biochem. Soc. Trans. 2002, 30, 1047-1050. [CrossRef] [PubMed]

32. Sun, T.; Hayakawa, K.; Bateman, K.S.; Fraser, M.E. Identification of the Citrate-Binding Site of Human ATP-Citrate Lyase Using X-ray Crystallography. J. Biol. Chem. 2010, 285, 27418-27428. [CrossRef] [PubMed]

33. Fabiszewska, A.; Misiukiewicz-Stępień, P.; Paplińska-Goryca, M.; Zieniuk, B.; Białecka-Florjańczyk, E. An Insight into Storage Lipid Synthesis by Yarrowia lipolytica Yeast Relating to Lipid and Sugar Substrates Metabolism. Biomolecules 2019, 9, 685. [CrossRef] [PubMed]

34. Lu, S.; Wang, J.; Chitsaz, F.; Derbyshire, M.K.; Geer, R.C.; Gonzales, N.R.; Gwadz, M.; Hurwitz, D.I.; Marchler, G.H.; Song, J.S.; et al. CDD/SPARCLE: The Conserved Domain Database in 2020. Nucleic Acids Res. 2020, 48, D265-D268. [CrossRef] [PubMed]

35. Goodwin, T.W. [29] Biosynthesis of Carotenoids: An Overview. In Methods in Enzymology; Elsevier: Amsterdam, The Netherlands, 1993; pp. 330-340.

36. Li, L.; Liu, Z.; Jiang, H.; Mao, X. Biotechnological Production of Lycopene by Microorganisms. Appl. Microbiol. Biotechnol. 2020, 104, 10307-10324. [CrossRef] [PubMed]

37. Albertyn, J.; Pohl, C.H.; Viljoen, B.C. Rhodotorula. In Encyclopedia of Food Microbiology; Elsevier: Amsterdam, The Netherlands, 2014; pp. 291-295.

38. Buzzini, P.; Innocenti, M.; Turchetti, B.; Libkind, D.; van Broock, M.; Mulinacci, N. Carotenoid Profiles of Yeasts Belonging to the Genera Rhodotorula, Rhodosporidium, Sporobolomyces, and Sporidiobolus. Can. J. Microbiol. 2007, 53, 1024-1031. [CrossRef] [PubMed]

39. Heath, R.J.; Rock, C.O. The Claisen Condensation in Biology. Nat. Prod. Rep. 2002, 19, 581-596. [CrossRef] [PubMed]

40. Chevillard, G.; Clémencet, M.-C.; Latruffe, N.; Nicolas-Francès, V. Targeted Disruption of the Peroxisomal Thiolase B Gene in Mouse: A New Model to Study Disorders Related to Peroxisomal Lipid Metabolism. Biochimie 2004, 86, 849-856. [CrossRef]

41. Guo, W.; Tang, H.; Zhang, L. Lycopene Cyclase and Phytoene Synthase Activities in the Marine Yeast Rhodosporidium Diobovatum Are Encoded by a Single Gene CrtYB: Lycopene Cyclase/Phytoene Synthase of Rhodosporidium diobovatum. J. Basic Microbiol. 2014, 54, 1053-1061. [CrossRef] [PubMed]

42. Pham, K.D.; Shida, Y.; Miyata, A.; Takamizawa, T.; Suzuki, Y.; Ara, S.; Yamazaki, H.; Masaki, K.; Mori, K.; Aburatani, S.; et al. Effect of Light on Carotenoid and Lipid Production in the Oleaginous Yeast Rhodosporidium Toruloides. Biosci. Biotechnol. Biochem. 2020, 84, 1501-1512. [CrossRef]

43. Pan, X.; Wang, B.; Duan, R.; Jia, J.; Li, J.; Xiong, W.; Ling, X.; Chen, C.; Huang, X.; Zhang, G.; et al. Enhancing Astaxanthin Accumulation in Xanthophyllomyces Dendrorhous by a Phytohormone: Metabolomic and Gene Expression Profiles. Microb. Biotechnol. 2020, 13, 1446-1460. [CrossRef]

44. Dhar, H.; Chaudhary, A.; Rana, V. Designer Microbes for Nutraceutical Application. In Advances in Agri-Food Biotechnology; Springer: Singapore, 2020; pp. 239-285.

45. Chamovitz, D.; Sandmann, G.; Hirschberg, J. Molecular and Biochemical Characterization of Herbicide-Resistant Mutants of Cyanobacteria Reveals That Phytoene Desaturation Is a Rate-Limiting Step in Carotenoid Biosynthesis. J. Biol. Chem. 1993, 268, 17348-17353. [CrossRef]

46. Guo, W.; Liu, Y.; Yan, X.; Liu, M.; Tang, H.; Liu, Z.; Zhang, L. Cloning and Characterization of a Phytoene Dehydrogenase Gene from Marine Yeast Rhodosporidium Diobovatum. Antonie Leeuwenhoek 2015, 107, 1017-1027. [CrossRef] [PubMed]

47. Nekrutenko, A.; Lariviere, D.; Rasche, H. Making Sense of a Newly Assembled Genome (Galaxy Training Materials). 2020. Available online: https://training.galaxyproject.org/trainingmaterial/topics/assembly/tutorials/ecoli_comparison/tutorial html (accessed on 3 December 2020). 